\title{
Mitral Regurgitation Classification using MrNetDeep Learning Model
}

\author{
Vishal Chandra ${ }^{1}$, Prattay Guha Sarkar ${ }^{2}$, Vinay Singh $^{3}$ \\ ${ }^{1}$ Usha Martin University, India, vcvishalchandra@gmail.com \\ ${ }^{2}$ Rajendra Institute of Medical Sciences, India, prattay_2904@yahoo.com \\ ${ }^{3}$ Usha Martin University, India, vinaysinghuma@gmail.com
}

\begin{abstract}
Cardiovascular diseases are the major cause of disabilities and the responsibility for the death in underdeveloped countries. Unless and until end-stage reached Mitral Regurgitation (MR) is does not show any symptoms, the most common vascular disease. Thus, it is very crucial to diagnose in its early stage and start proper treatment to prevent it to go worse stage. 2D echocardiography is the most common modality for assessing the severity of MR. For diagnosis of severity of mitral regurgitation, echocardiography Is one common modality. One of artificial intelligence technique called deep learning deals with images and videos helpful in borderline cases for echocardiographers. In this paper, we proposed a lightweight deep learning model MrNetwhich can able to classify of MR such as moderate, mild moderate of MR is based MR clips of echocardiographic to assists sonographers at the first line of diagnosis. Detection criteria are used, the color of the regurgitant jet(blue) inside Left Atrium, in sever and moderate MR, the jet is easily visible but in some cases of mild MR, it may misdiagnose. Our proposed model was successfully able to classify MR and normal cases images whether it is mild, moderate and severe. Our proposed model consists of the Normal convolution and ResNet hybrid model. The aim is to use the minimum parameter to achieve real-time detection. there is a pattern present in mitral regurgitation and non-regurgitation, which is jet. the methodology is based upon the color pattern of MR the best accuracy achieved in mild $96 \%$, moderate $94 \%$ and sever $90 \%$ and matrices used are classification report and confusion matrix.
\end{abstract}

Key words: Mitral regurgitation, Deep learning, detection. 2D echocardiography, abnormality.

\section{INTRODUCTION}

The developed countries, as well as underdeveloped countries, are suffered from the second most common cardiac valvular diseases is MR. [1] and so for the treatment process point of view, early diagnosis is crucial. MR is a happens in which due to incomplete closer of Mitral valve blood flows to reverse direction which left atrium from left ventricle during systole [2], and MR has two types primary and secondary. patients having MR feel decreased exercise tolerance and dyspnoea [3]. MR can be detected by a stethoscope by patients' physical examination, having early diastolic rumble can be observed, loud systolic murmur in third heart sound. For the assessment of MR 2D color, Doppler Echocardiography best and cheapest methods [4]. Vena contracta(VC), effective regurgitant orifice(ERO), and regurgitant volume are the distinct criteria guided by THE American College of Cardiology for and American Heart Association(ACC/AHA) . assessment of the severity of MR by color Doppler echocardiography [3]. for the estimation of the mitral regurgitant, orifice $\mathrm{VC}$ (vena contracta) is an one of the important parameter because it is the narrowest place of regurgitant. while taking echocardiographic view, the narrowest part at the mitral valve level orthogonal to coaptation line, of the MR jet is VC. there are two best way to capture VC A4C(apical four chamber) view and PLAX(parasternal long axis view. some times A4C gives falsely increase the VC and PLAX provide best view for determination of VC. For the calculation of ERO, the proximal isovelocity surface area (PISA) must be measured accurately for the determination of the severity of MR [5]. During the mid-systole, the frame of A4C view PISA for the calculation of ERO.

Since the ERO important (effective regurgitant orifice) is estimate by the PISA(proximal isovelocity surface area) is radius of mid-the systolic is produced can be see $\mathrm{A} 4 \mathrm{C}$ view. By for the assessment of MR severity one of the best method is ERO calculation. To visualize and measure smallest width distal of regurgitant is $\mathrm{VC}$. the $\mathrm{VC}$, we should measure the smallest width just distal to the regurgitant orifice [4]. for MR severity estimating the mitral regurgitation is has guideline of the latest AHA/ACC guideline for MR of $\mathrm{r} \mathrm{VC}$ and ERO as follows as mild: VC o 0.3; Moderate: 0.3 o VC o 0.7, ERO o 0.4, Regurgitant volume $60 \mathrm{ml}$; Severe: VC 4 0.7, ERO 4 0.4, Regurgitant volume $460 \mathrm{ml}$ ) [3].

Additionally, for the severity diagnosis of mitral regurgitation other useful technology CMR (cardiac magnetic resonance), too much in recent years it utilises has increased in [6]. Thavendiranathan et al. systematic reviewed the evaluation conducted, which is a accurate and responsible technology to the severity quantification of MR. The research result shows 
cardiac CT scan, CMR ,3D echocardiography and technologies are superior to other technologies [7].

2D Echocardiography, mostly, commonly, cost-effective and non-invasive technology for the severity of MR assessment and so therefore in our paper, we have chosen 2D echocardiography for automatic classification the of MR mild, moderate and severe, and in the coming sections, we briefly explain the echocardiography images and literature review.

In heart diseases for the first line of defence is echocardiography since it has following features available technology for the diagnosis of heart diseases it is first line defence. since its non-invasive nature of examination, it is the best method Echocardiography is one of the best method due to its non-invasive nature of nature of working [8]. for the visualization of the heart and heart functions TTE (Transthoracic echocardiograms) by putting the transducer on the chest wall which gives the details of heart's functioning's. for the visualization of apical four chamber view (A4C) view sonographer has to place the transducer to the apex of the heart.to analysis of different sections which are from base to apex used, captured image and clips are not only low resolution which makes decision difficult. Therefore, computer-aided diagnosis (CAD) is helpful for cardiologists, especially in borderline cases. for the visualization of apical 2 chamber view(A2C), transducer has to rotate clockwise.

If the transducer is rotated $90^{\circ}$ clockwise, the apical 2-chamber (A2C) view will be visible. The parasternal short axis (PSAX) view, which analyzes the different sections of the heart from base to apex, is captured by rotating the transducer 90 degrees clockwise in the parasternal long-axis position [9]. Analysis of the echocardiography images by cardiologists is very important, but echocardiography images are low resolution with artifacts that make the decision difficult. hence, artificial intelligence helpful for cardiologists, which works automically especially in borderline cases.

\section{LITERATURE REVIEW}

Due to use of artificial intelligence techniques there have improvement in diagnosis of heart diseases in last decade. there are two most famous and useful way to use artificial intelligence are machines learning and deep learning for detection. deep learning deals with images and videos, there for in our research deep learning. hybrid Hidden Markov((HMM-SVM), Support Vector Machine(SVM) model gives cyclic time series [10]; a neural network of multi-layer feed-forwarded used data fusion method to classics heart diseases [11]; for the diagnosis of coronary artery using a fuzzy-par- particle swarm optimization (fuzzy-PSO) model used[12]; for diagnosis of ventricular septal defect, the growing time support vector machine, of innocent and pathological murmurs [13]; and the support vector machine classifiers diagnosis of the ventricular septal defect (VSD) [14] they are the examples of these researches on heart diseases. Hanbay [15] uses Doppler heart sounds
(DHS) data with a Least Square Support Vector Machine (LS-SVM) classifier and wavelet packet decompositions (WPD) and Fast Fourier Transform (FFT) as features for the classification of heart valve diseases. The reported accuracy for two normal and abnormal groups which consists of MR subjects is 96.13\%. Maglogiannis et al. [16] use DHS data with wavelet decompositions and Short Time Fourier transform (STFT) as features with the SVM classifier for the classification of heart valve disease. The reported accuracy for two normal and abnormal groups is $91.43 \%$, for aortic stenosis (AS) and the MR classification, is $91.67 \%$

and, for aortic regurgitation (AR) and mitral stenosis (MS), the classification is $93.42 \%$.

Furthermore, to date, there have not been any reports on the automatic diagnosis of the severity of MR. Also, color Doppler echocardiography is a more complex method than 2Dechocardiography to implement, and its results are operator dependent [14]. Thus, we present a novel method to detect the severity of MR in 2D echocardiography and to classify MR patients from normal subjects with a high degree of accuracy.

\section{DATA COLLECTION}

All have collected from cozy care hospital under-informed informed consent we collected echocardiography view of A4C(apical four-chamber) view of 86 clips(70 for training and 16 for testing) of mid systolic view. 70 for mild, 70 for moderate and 70 sever,16, mild, 16 moderate and 16 for testing of Mitral regurgitation patients. All clips are in avi format having $30 \mathrm{fps}$ (frame per second) using siemens machine. clips are chosen for training and testing blindly. no pre-processing has done. image size used is $512 * 512$, images are normalized by 0,255 .

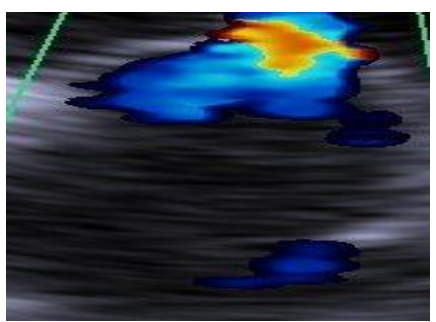

MILD

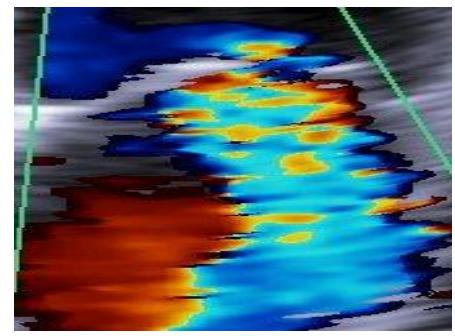

SEVERE

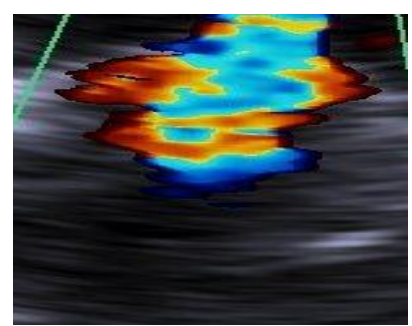

MODERATE

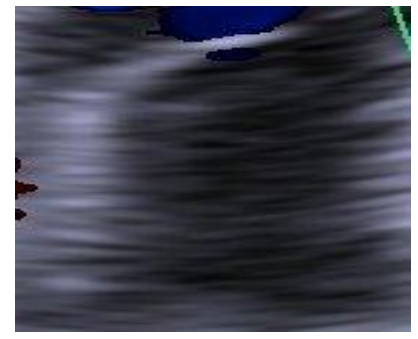

NORMAL
Figure 1: Different color doppler for mitral valve. 
Vishal Chandra et al., International Journal of Advanced Trends in Computer Science and Engineering, 9(2), March - April 2020, 1574 - 1582

\section{ResNet 18 ARCHITECTURE}

We used a modified ResNet model which more efficient and has a smaller number of parameters, makes it a real-time application. We used both $\mathrm{L} 1$ and L2 regularization.

I. In 2015 it won ILVRC classification competition with an error rate of $3.57 \%$ (An ensemble model).

II. It won the 1st prize in COCO 2015 competition in ImageNet Detection COCO detection ImageNet localization and COCO segmentation.

III. It replaced VGG-16 layers in Faster R-CNN with ResNet-101.Replacing VGG-16 layers in Faster R-CNN with ResNet-101. it gave an improvement of $28 \%$.

IV. The network trained with 100 layers and 1000 layers.

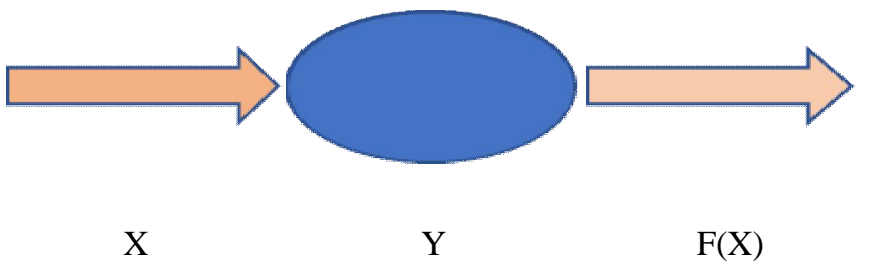

Figure 2: Plain block

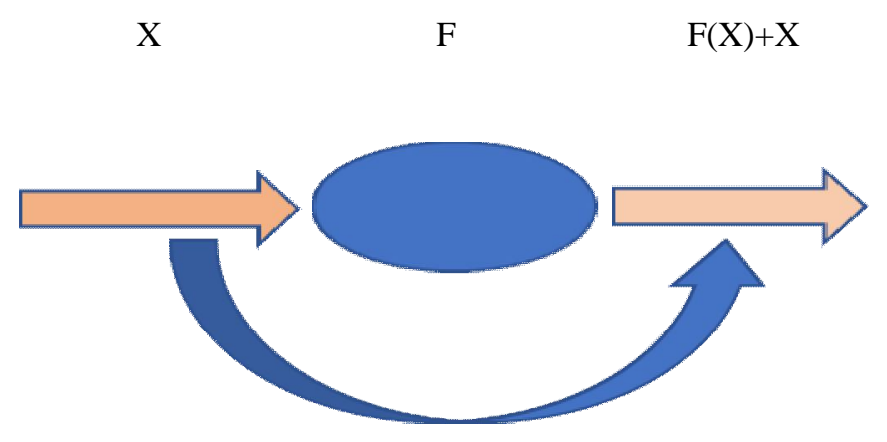

Figure 3: Residual block
Table 1: Dilated $18 * 2$

\begin{tabular}{|l|l|l|}
\hline Layer Name & Output Size & 18 -Layer \\
\hline Conv 1 & $512 * 512$ & {$[3 * 3,64]^{* 2}$} \\
\hline Conv2_x & $128^{*} 128$ & {$[3 * 3,128]^{* 2}$} \\
\hline $\begin{array}{c}\text { Conv3_x(dilation } \\
\text { rate=2) }\end{array}$ & $64 * 64$ & {$[3 * 3,64]^{* 2}$} \\
\hline Conv4_x & $32 * 32$ & {$[3 * 3,32]^{* 2}$} \\
\hline Conv5_x & $16 * 16$ & {$[3 * 3,16]^{* 2}$} \\
\hline & $1 * 1$ & $1.8 * 10^{9}$ \\
\hline FLOPs & & \\
\hline
\end{tabular}

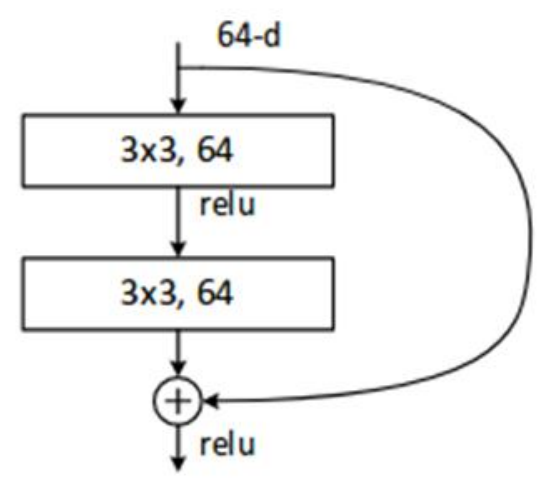

Figure 4: Identity block of general Resnet model

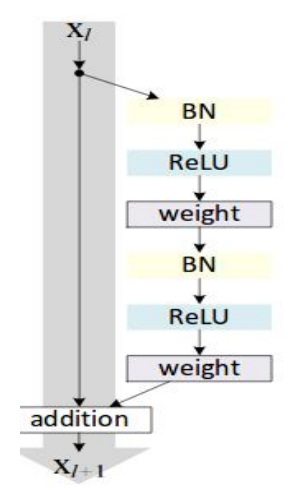

full pre-activation

Figure 5: Resnet identity module 
Vishal Chandra et al., International Journal of Advanced Trends in Computer Science and Engineering, 9(2), March - April 2020, 1574 - 1582

\section{Why ResNet is different and useful?}

\section{The major problem is:}

When deeper networks start converging, degradation has been exposed when deeper networks start converging., accuracy getting results to degrade rapidly when saturation occurred.

\section{PROPOSED MODEL}

Our proposed model which is also known as MrNetcapable of classification of MR (mitral regurgitation) in the heart which between the left atrium and left ventricle.

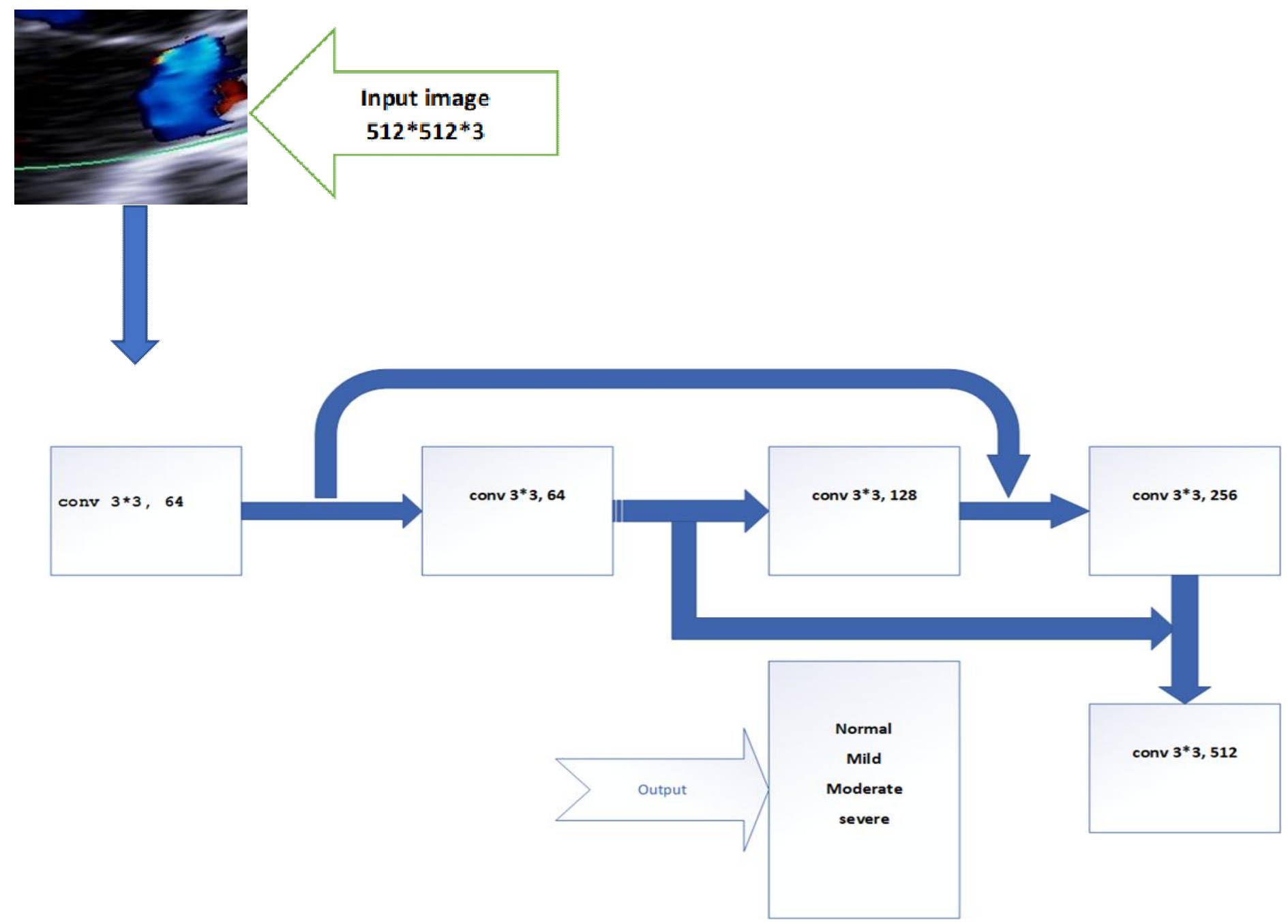

Figure 6: Proposed model 
The proposed model is inspired by the ResNet model, each identical module consists of as figure 5, the last layer is the avg pooling layer, every module has two activation functions ReLU, two batch normalization, and two CNN. the last layer is the SoftMax layer which classifies mild, moderate, normal and severs output. There are two skip connections. every module has $3 * 3$ kernel size and filter size increasing 64 to 512 by twice in each module, having only 4,907,393 of trainable parameters.

\section{RESULTS}

Matrices for mild used in our research has accuracy, sensitivity, mcor, specificity, recall, precision, f1, having, evaluation values are

for Mild

$0.5056082606315613, \quad 0.96875, \quad 0.9375$, $0.9393364191055298,0.9359828233718872,0.9375,1.0$, 0.967741847038269

for Moderate

$0.75,0.774596631526947,0.9979164600372314,0.75,1.0$, 0.8571428060531616 , respectively, and

for Severe

$0.5220469236373901,0.90625,1.0,0.827170193195343$, $0.684420645236969, \quad 1.0, \quad 0.8421052694320679$, 0.9142856597900391

\subsection{Confusion Metrics}

MILD

16

0

1

Figure 7.Mild and normal
MODERATE

15

7

Figure 8.Moderate and normal

SEVERE

16

0
NORMAL

1

9

$\begin{array}{cc}\text { SEVERE } & \text { NORMAL } \\ 16 & 0 \\ 0 & 16 \\ \end{array}$

Figure 9: Severe and normal

$\begin{array}{lllll} & \text { PRECISION } & \text { RECALL } & \text { F1-SCORE } & \text { SUPPORT } \\ \text { NORMAL } & \mathbf{1 . 0 0} & \mathbf{0 . 5 0} & \mathbf{0 . 6 7} & \mathbf{1 6} \\ \text { SEVERE } & \mathbf{0 . 6 7} & \mathbf{1 . 0 0} & \mathbf{0 . 8 0} & \mathbf{1 6} \\ \text { MODERATE } & \mathbf{0 . 7} & \mathbf{0 . 5 6} & \mathbf{0 . 8 2} & \mathbf{1 6} \\ \text { MILD } & \mathbf{1 . 0 0} & \mathbf{0 . 8 8} & \mathbf{0 . 9 3} & \mathbf{1 6} \\ \text { ACCURACY } & & & \mathbf{0 . 7 8} & \mathbf{3 2} \\ \text { (MODERATE) } & & & & \\ \text { ACCURACY } & & & \mathbf{0 . 9 4} & \mathbf{3 2} \\ \text { (MILD) } & & & & \\ \text { ACCURACY } & & & \mathbf{0 . 9 4} & \mathbf{3 2} \\ \text { (SEVERE) } & & & & \end{array}$

CLASSIFICATION REPORT 


\subsection{Output Images}

We represented infected as Mild,Moderate and Severe are infected and uninfected is Normal respectively.
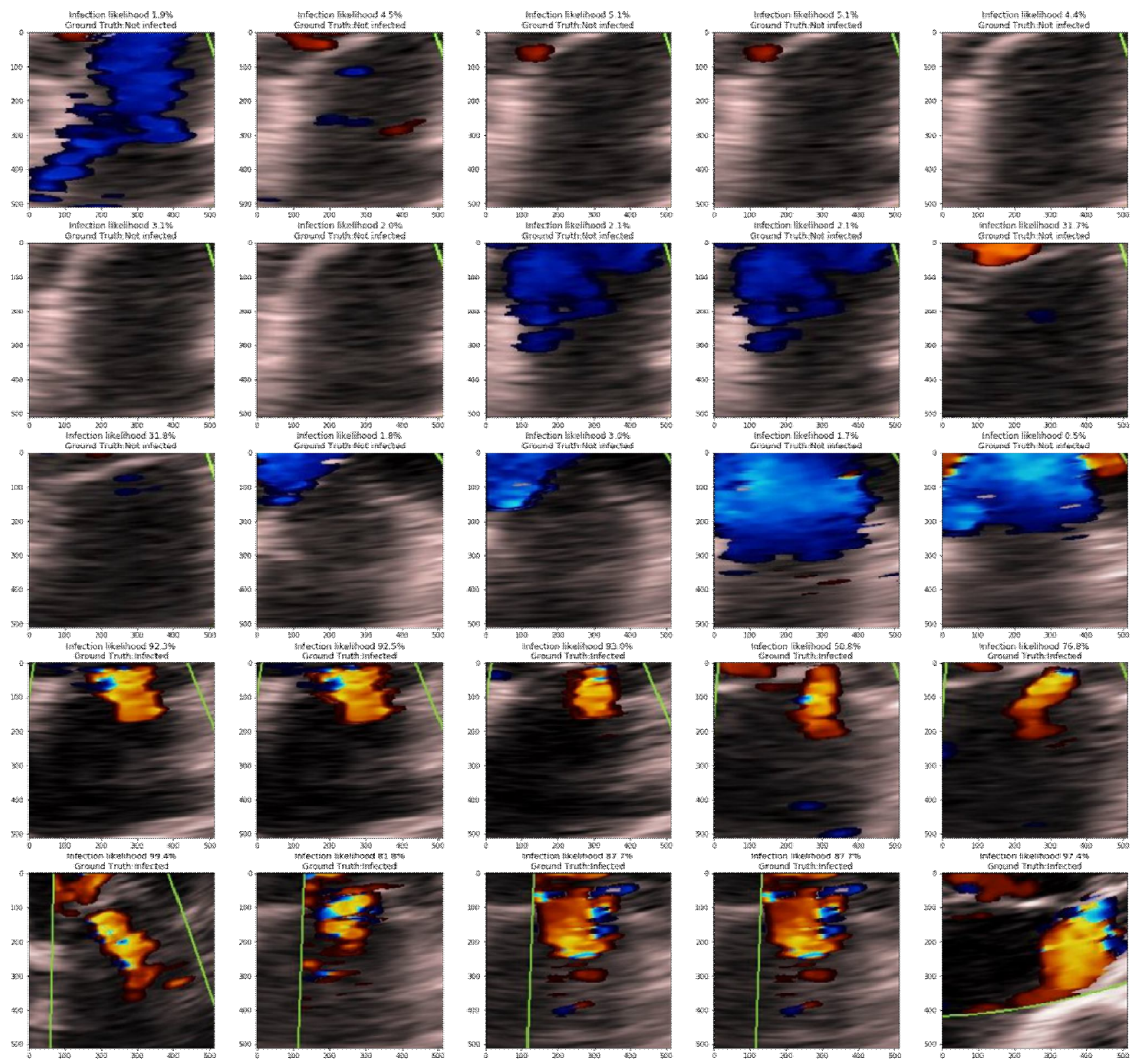

Figure 10: Mild and Normal 

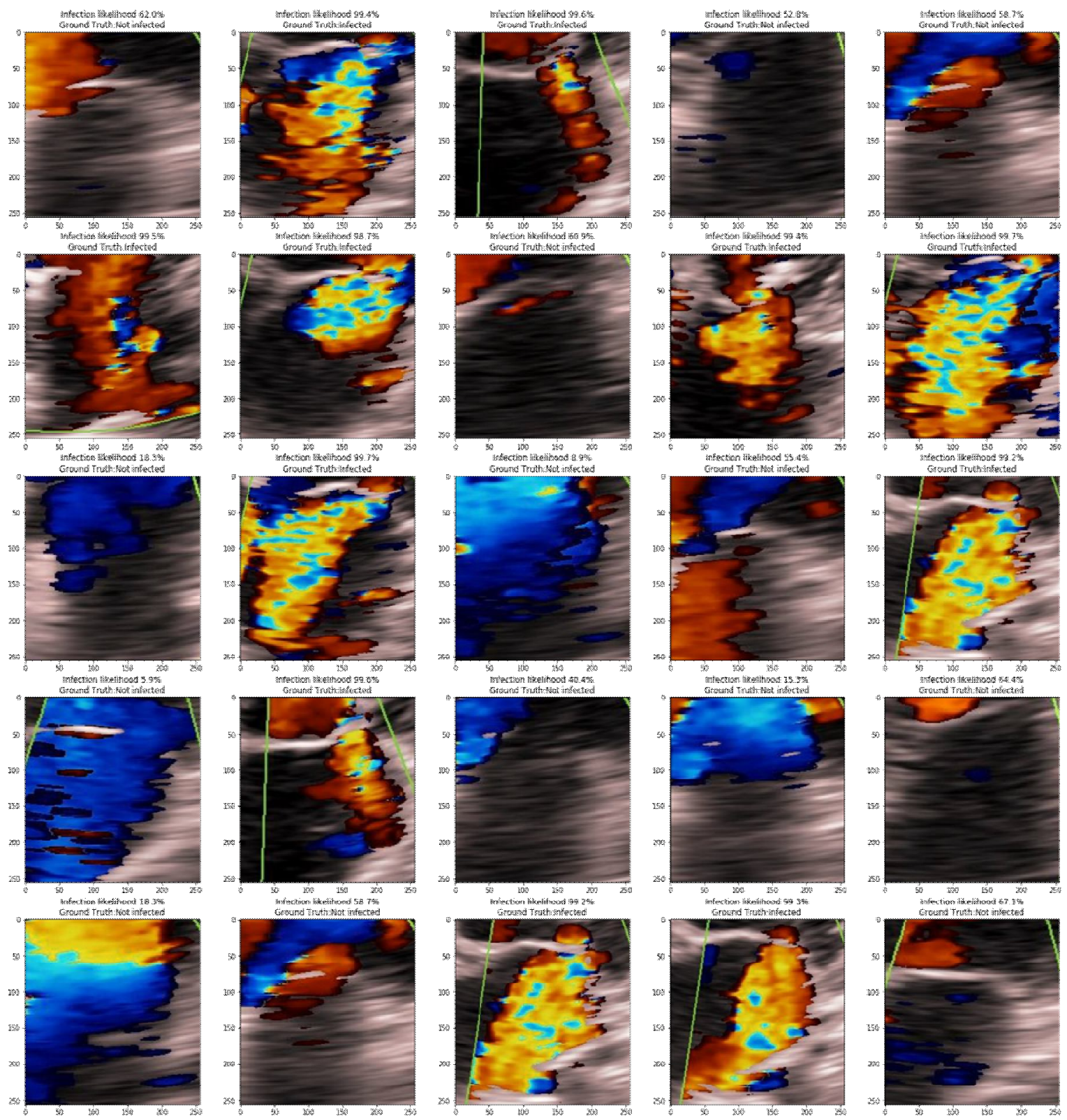

Figure 11: Moderate and Normal 

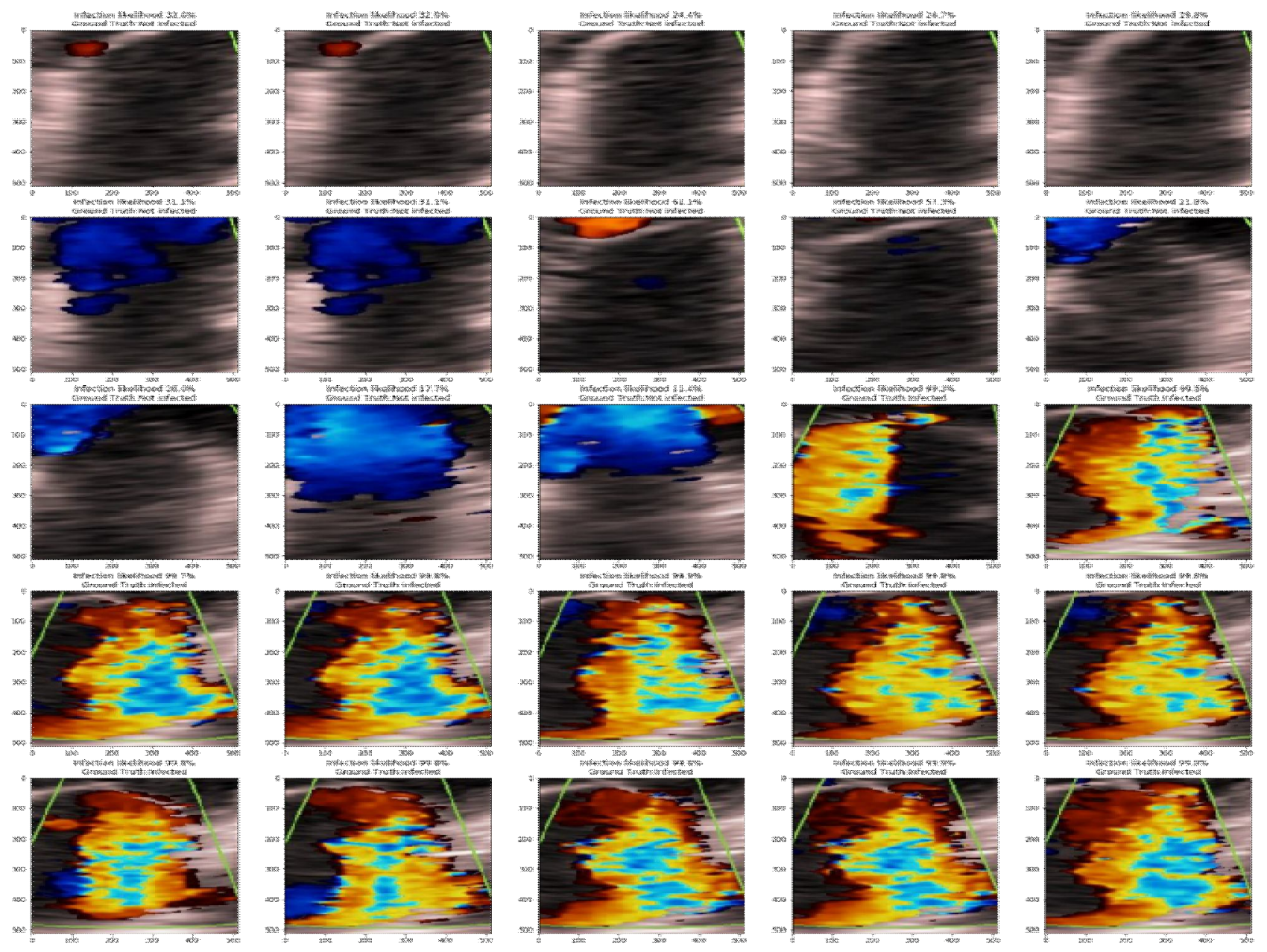

Figure 12: Severe and Normal

\section{CONCLUSION}

As for results it easily to see that mild samples are classified almost perfectly, classify even in the small dataset. also, our proposed model has a smaller number of parameters which will make a real-time expert system. our proposed model named MrNet, which can classify Mitral regurgitation has a good extend. we can trust MrNetto detect automatically MR in mild, moderate, normal and severe cases. It will help echocardiographer for detection MR (mitral regurgitation) automatically. In this way, consumption of time will be reduced and more and more patients will be able to serve. which helps in under developing countries where time is most important.

\section{REFERENCES}

[1]B.Iung,et al., A prospective survey of patients with valvular heart disease in Europe: the euro heart survey on valvular heartdisease, Eur.HeartJ.24(13) (2003)1231-1243. https://doi.org/10.1016/S0195-668X(03)00201-X

[2]S.Gelsomino,et al., Left ventricular diastolic functional after restrictive mitral ring annuloplasty in chronic ischemic mitral regurgitation and its predictive value on outcome and recurrence of regurgitation, Int.J.Cardiol.132(3) (2009)419-428.

https://doi.org/10.1016/j.ijcard.2007.12.058

[3]R.A.Nishimura, et al., AHA/ACC guideline for the management of patients with valvular heart disease: executive summary: are port of the American College of 
Cardiology/American Heart Association Task Force on Practice Guidelines, Circulation 129(23)(2014)2440.

[4]W.F.Armstrong, T.Ryan, $\quad$ Feigenbaum's Echocardiography, Lippincott Williams \& Wilkins, UnitedStates, 2012.

[5] X. Zeng,etal.,Echocardiography of the mitral valve,Prog.Cardiovasc.Dis.57 (1) (2014)55-73

https://doi.org/10.1016/j.pcad.2014.05.010

[6]S.Kar,R.Sharma, Current assessment of mitral regurgitation :not making the grade*, J.Am.Coll.Cardiol.65(11)(2015)1089-1091.

https://doi.org/10.1016/j.jacc.2015.02.001

[7]P.Thavendiranathan,etal., Quantitative assessment of mitral regurgitation: validation of new methods,J.Am.Coll.Cardiol.60(16)(2012)1470-1483.

[8]T.Binder,et al., Artificial neural networks and spatial temporal contour linking for automated endocardial contour detection one chocardiograms: anovel approach to determine left ventricular contractile function, Ultrasound Med. Biol. 25(7)(1999)1069-1076.

[9]N.Quader,V.H.Rigolin, Two and three dimensional echocardiography for pre- operative assessment of mitral valveregurgitation,Cardiovasc.Ultrasound 12 (1) (2014)42.

[10]A.Gharehbaghi, P.Ask, A.Babic, A pattern recognition framework for detecting dynamic changes on cyclic time series, Pattern Recognit.48(3)(2015) 696-708.

https://doi.org/10.1016/j.patcog.2014.08.017

[11]M.Obayya, F.Abou-Chadi, Data fusion for heart diseases classification using multi-layer feed forward neural network, in: Proceedings of IEEE International Conference on Computer Engineering \& Systems,ICCES,2008.

[12]N.G.Hedeshi, M.S.Abadeh, Coronary artery disease detection using a fuzzy- boosting PSO approach,Comput.Intell.Neurosci.2014(2014)6. https://doi.org/10.1155/2014/783734

[13]A.Gharehbaghi,etal., A novel method for discrimination between innocent and pathological heart murmurs, Med.Eng.Phys.(2015).

https://doi.org/10.1016/j.medengphy.2015.04.013

[14]S.Sun,et al., Segmentation-based heart sound feature extraction combined with classifier models for a VSD diagnosis system,ExpertSyst.Appl.41(4) (2014)1769-1780. https://doi.org/10.1016/j.eswa.2013.08.076 